\title{
XV.
}

\section{Ueber Hallucinationen und Gedankenlautwerden ${ }^{1}$ ).}

\author{
Von \\ Dr. Döllken, \\ Privatdocenten in Leipzig.
}

\begin{abstract}
Trotz des grossen Interesses, welches die Hallucinationen immer für den Psychiater gehabt haben, sind die Publicationen über uncomplicirte hallucinatorische Zustände und deren Analyse ziemlich selten. Dabei kommen derartige Zustände relativ bäufig zur Beobachtung. Probst ${ }^{2}$ ) behauptet dasselbe und beschreibt sehr eingehend einen Einzelfall. Uh th off ${ }^{3}$ ) analysirt eine Reihe von Fällen mit uncomplicirten Gesichtshallucinationen. Ich selbst konnte im Verlauf einiger Jahre 11 Fälle von Hallucinose, die nicht mit Geisteskrankheit complicirt war, beobachten. Allerdings kommen derartige Kranke selten in die Klinik oder Anstalt, sondern bleiben in der Behandlung des "Nervenarztes". Viele von ihnen haben eine Scheu, von ihren Hallucinationen zu sprechen. Andere (meist Belastete oder Hysterische) balten diese Erscheinung für so selbstverständlich normal, dass nur eine störende Steigerung des Symptoms oder ein zufälliges Nachforschen dem Axzt die Kentniss bringt. Es ist klar, dass ein Material, bei dem wir nicht mit Wahnideen, Bewusstseinsstörungen und Sejunction etc. zu rechnen haben, in vieler Beziehung den klareren Einblick in den Mechanismus der Hallucinationen und in ihre Werthigkeit zulässt.
\end{abstract}

Auffallend ist es, wie von vielen Autoren die Grösse des betheiligten Leitungsbogens unterschätzt wird.

1) Vortrag, gehalten auf der Versammlung mitteldeutscher Psychiater und Neurologen am 27. October 1907 in Leipzig.

2) Probst, Veber das Gedankenlautwerden und über Hallucinationen ohne Wahnideen. Monatsschr. für Psych. und Neurol. Bd. XIII. 1903.

3) Uhth off, Beiträge zu den Gesichtstäuschungen bei Erkrankungen des Sehorgans. Monatsschr. für Psych. und Neurol. 1899. 
Nicht befriedigen können die Theorien, welche versuchen, den Mechanismus der Hallucinationen in eine einheitliche Formel za bringen. So passt weder die Theorie der primären Reizung des Erinnerungsbildcentrums, noch die Suggestionstheorie Jendrassik's ${ }^{1}$; auf alle analysirbaren Fälle. Wernicke $e^{2}$ meint, dass in Folge des Sejunctionsvorgangs zunächst bestimmte mọtorische (oculomotorische etc.) Erinnerungsbilder erregt werden und erst von da aus - rückläufig - die zugeordneten percipirenden Elemente der Sinnesfelder (Lichtfeld etc.). Diese sind bei dem Vorgang im Wesentlichen die Träger des Organgefühls. Auch diese Erklärung lässt in sehr vielen concreten Fällen völlig im Stich.

Die hallucinirenden Taubstummen Cramer's 3 ) zeigen deutlich, dass auf verschiedenem Wege - entweder Sprachbewegungsbilder der Zunge, des Kehlkopfes etc. oder der Hände - die hallucinatorischen Wortbilder am leichtesten ansprechbar sein können.

Verschiedenartige Ausprechbarkeit eines Centrums, je nachdem der. eine oder andere Weg gewählt wird, findet sich bekanntermaassen auch in der Norm, und zwar als Disposition und tritt schon in frühem Lebensalter in Erscheinung. Der eine Sextaner lernt seine Vocabeln hauptsächlich durch den optischen Eindruck, der andere durch Sprechbewegungsbilder. Einer meiner Mlitschüler entdeckte erst als Secundaner das System, wie er am besten Vocabeln lernen konnte! Zuhülfenahme von markirenden Sehreibbewegungen. Die neuerdings beliebteste Methode, sehr vorwiegend durch die gesprochenen Wortbilder eine fremde Sprache zu erlernen, ist sicher für Viele geeignet. Ich selbst aber war von jeher trotz mehrfacher ernster Versuche mit verschiedeneu Sprachen nicht im Stande, rasch vorwärts zu kommen, wenn ich nur die Wortklangbilder aufnebmen konnte, dagegen gelang es ohne jede Mühe mit Zuhülfenabme der visuellen Wortbilder (motorischer, akustischer, visueller Typus).

Derartige Ueberlegungen machen es von vornherem schon unwahrscheinlich, dass Hallucinationen immer nach demselben starren Schema entstehen müssen.

Allgemein anerkannt ist, dass die Hallucinationen in den corticalen

1) Jendrassik, Ueber die Entstehung der Hallucinationen und des Wahns. Neurol. Centralbl. 1905.

2) Wernicke, Grundriss der Psychiatrie. 1900. (Dort auch S. $198 \mathrm{ff}$. eine Darstellung älterer Theorien.)

3) Cramer, Die Hallucinationen des Muskelsinnes. Freiburg 1889. Derselbe, Ueber Sinnestäuschungen bei geisteskranken Taubstummen. Archiv für Psych. Bd. XXVIII. 
Projectionscentren manifest werden, ihre Plasticität erlangen und dass die zugehörigen Erinmerungscentren in erster Linie mitwirken müssen.

Flechsig ${ }^{1}$ ) spricht sich dahin aus, dass die Erregung von den Assiationscentren rückläufig auf verschiedene Sinnescentren einwirken kann. In den weueren Lehrbüchern sind Ziehen, Wernicke, Kraepelin, Binswanger und Siemerling, Sommer u. A. der Meinung, dass die hallucinatorische Reizung eine rückläufige Richtung nimmt:

Dagegen erheben sich in letzter Zeit wieder Stimmen wie Jendrassik; Störring ${ }^{2}$ ) a. A. mehr oder minder gegen eine centrifugale Leitung in centripetalen Bahnen.

In der That giebt es einzelne Fälle, welche die Erklärung zulassen, dass die Hallucinationen auf dem üblichen Wege in der normalen Richtung der Sinnesleitung - centripetal - laufen. In den weitaus meisten Fällen jedoch finde ich keinen Anhaltspunkt für diese Deutung.

An sich ist eine centrifugale Leitung in centripetalen Bahnen durchaus nicht unerhört. Im peripheren Nerven ist sịe bereits von v. Dubois-Reymond bewiesen und im Räckenmark nicht unwahrscheinlich $\left[\right.$ [Kohnstam m ${ }^{3}$ )].

Damit eine Hallucination zu Stande kommen kann, müssen bestimmte Bahnen und Centren überregbar gemacht, gewissermaassen activirt werden. Die Activirung erfolgt durch einen pathologischen Process oder es handelt sich um reizbare Schwäche eines oder mehrerer Leitungsbogen. Letzteres ist meist angeborene Aulage.

Die Activirung kann auf verschiedenen Wegen und durch verschiedene Mittel erfolgen:

1. Angeborene Uebererregbarkeit eines Leitungsbogens.

2. Erkrankung eines peripheren Organs, Neuritis, Neuralgie, Otitis etc. activirt vom Endorgan und peripheren Nerven aus.

Wernicke hält es für unwahrscheinlich, dass vom peripheren Nerven aus Hallucinationen ausgelöst werden und führt als Beispiel Gesichtshallucinationen bei längst reizlosem, atrophischem Nervas opticus an. Es giebt aber Fälle, in denen sicher vom atrophirenden Nervus opticus die Activirung der Bahn erfolgt. Andererseits sind genug Fälle bekannt, in denen vom völlig reizlosen Opticus nach Jahren keine Activirung mehr erfolgen konnte. Es besteht nicht der mindeste Grund anzunehmen, dass derselbe Effect (die Gesichtshallucination) entweder nur central oder nur peripher enstehen könnte.

1) Flechsig, Gehirn und Seele. 1896.

2) Störring, Psychopathologie. Leipzig 1900.

3) Kohnstamm, Die centrifugale Leitung im sensiblen Endneuron. Zeitschr. für Nervenheilk. Bd. XXI. 
3. Activirung von subcorticalen Centren aus. Flechsig's ${ }^{1}$ ) Fall von Kalkconcrementen im hinteren Vierbügel. Weinland's ${ }^{2}$ ) Fall (Laboratorium Flechsig) eines Tumors im vorderen Vierhügel.

4. Activirung von centralen Läsionsstellen aus. Hallucinationen in gelähmten Gliedern nach Apoplexie im Bereich der centralen motorischen Bahn, aphasiscbe, hemianopische Hallucinationen [Pick, $\left.\left.\mathrm{Hoche}^{3}\right)\right]$.

5. Central angreifende Gifte, Opium, Atropin, Haschisch, Santonin, Hyoscin, Schwefelkohlenstoff, Blei etc. können Hallucinationen hervorrufen.

6. Höchst wahrscheinlich haben auch die meisten Bacterien- und Stoffwechselgifte rein centrale (wenn auch diffuse) Angriffspunkte ${ }^{4}$ ).

1. Bö., Schriftsetzer, 26 Jahre alt, nicht belastet, früher nie krank. Juni 1904 Angina und Neuralgie des linken Nervas frontalis und Nervus occipitalis minor. Während diese in etwa 10 Tagen verschwunden waren, blieben gleichzeitig aufgetretene acustische Erscheinungen noch 3 Monate bestehen. Ohrenbefund stets negativ, ein leichter Tubenkatarrh bestand kurze Zeit. Im Anfang hörte er das deutliche lante Sausen von Maschinen, wie sie in seinem Arbeitsraum standen, ausserdem noch "Klucksen". Nach etwa 14 Tagen bestand fast ununterbrochen noch das Klucksen. Aergerte er sich bei der Arbeit oder zuhause, hörte er 1-2 Tage lang wieder Maschinensausen. Dass es sich um Trugwahrnehmungen handelte, war dem Patienten stets klar. Im Maschinenraum konnte or das "Sausen im $\mathrm{Obr}^{\text {" }}$ rom gleichartigen Sausen seiner Maschine nicht unterscheiden. Dass es fortbestand, merkte er daran, dass bei plötzlichem Anhalten der Maschinen das "Sausen im $\mathrm{Ohr}$ allein weiter ging ${ }^{\text {" }}$. Galvanische Ströme 0,3 M.-A. von 3 Minuten Dauer unterdrückten die Hallucinationen, Ströme von 2 M.-A. verstärkten sie beträchtlich. Anode oder Kathode am $\mathrm{Ohr}$ war gleichgültig. Anoden- und Kathodenschliessung und-Oeffnung verursachen nur das gewöhnliche Knacken.

2. Ha., Schriftsetzer, 42 Jahre alt, nicht belastet, nie krank gewesen. Vorwiegend visueller Typus. December 1906 Influenza. Nach Ablauf der acuten Erscheinungen blieb „ein Sausen im Ohr, als wenn der Wind heftig durch Telephondrähte geht". Die specialärztlich sehr häufig vorgenommene Untersuchung der Obren ergab ausser einem leichten, bald geheilten Tubenkatarrh stets negativen Befund. Zeitweilig das Geränsch lant sausender Maschinen. Im Februar 1907 nach Anwendung von galvanischen Strömen 0,2 M.-A. nur ab und zu

1) Flechsig, Beziehungen des unteren Vierhügels zum Hörnerven. Neurol. Centralbl. IX.

2) Weinland, Archiv f. Psych. XXVI.

3) Pick, Hoche s. S. 444.

4) Siemerling, Artikel Amentia und Delirien in Binswanger-Siemerling's Lehrbuch. - Derselbe, Graviditäts- und Puerpera]psychosen. Deutsche Klinik. VI. 
Maschinensausen. In seinem Arbeitsraum verschwand das Geräusch völlig, wenn die die Maschinen liefen und stellte sich bald wieder ein, wenn abgestellt war. Als Patient nun im Mai 1907 eine Gemüthserregung hatte, trat das laute Geräusch windbewegter Telephondrähte wieder auf. Die Ohrenuntersuchung hatte negativen Befund. Patient war stets von dem subjectiven Charakter seiner Trugwahrnehmungen überzeugt. Da es ihn interessirte einen Vergleich zwischen den Trugwahrnehmungen und den Empfindungen von windbewegten Telephondrähten anzustellen, ging er mehrfach an windigen Tagen auf die Landstrasse. Es zeigte sich, dass die Hallucination mit dem wirklichen Geräusch eine Verschmelzung einging, so dass er sie nicht auseinander halten konnte. Enifernte er sich aber von den Drahtleitungen, blieb die Hallucination in derselben Intensität bestehen, während das Geräusch der Drähte schwächer wurde. Der Zustand besserte sich allmählich, besteht aber heute (nach 11 Monaten) noch fort. An Tagen niedrigen Luftdrucks sind die Gehörstäuschungen meist stark, an Tagen hohen Luftdrucks fast verschwunden. Anstrengungen, einige Glas Bier verschlimmern die Erscheinungen. Gemüthserregungen kommen bei dem Patienten kaum vor. Die Gefühlsbetonung der Hallucinationen ist sehr gering. Länger dauernde schwache galvanische Ströme 0,2 M.-A. vermögen die acuten Erscheinungen meist zu unterdrücken, stärkere galvanische von mehr als $2 \mathrm{M}$.-A. und ebenso mässig starke faradische und Wechselströme etwas zu steigern. Häufige Stromöffnung und -Schliessung steigert die Erscheinungen etwas.

In beiden Fällen ist die Activirung von der Peripherie her durch den Tubenkatarrh verursacht worden. Befallen ist der ganze centripetale Leitungsbogen von der Peripherie bis zum acustischen Erinnerungscentrum. ("Meine Maschinen"; Telephondrähte, die er in einer früheren Wohnung of hat sausen hören.) $\mathrm{Ob}$ der motorische Theil des Leitungsbogens in Mitleidenschaft gezogen ist, lässt sich nicht ergründcn. Motorische Erscheinungen wurden nicht einmal auf Suggestivfragen zugegeben.

Die Hallucinationen beider Patienten hatten starken Wirklichkeitscharakter. Sie wurden mit Hilfe der anderen Sinne corrigirt. Eine vollkommene Verschmelzung von subjectiver und objectiver Empfindung trat jedesmal bei einer gewissen Intensität des gleichlautenden äusseren Reizes ein und machte die Correctur unmöglich. Je nachdem also ein bestimmter Gehörsreiz angenähert oder entfernt wurde, erhielt die Hallucination objectiven Charakter oder nicht. Derartige Beispiele scheinen mir gegen Störring's (l. c.) Auffassung zu sprechen, dass eine haarscharfe principielle Unterscheidung zwischen Hallucinationen und Pseudohallucinationen gemacht werden kann.

3. Pe., Kaufmann, 33 Jahre alt. Vater am Schlaganfall gestorben, eine Schwester nervös. Die Familie ist sehr musikalisch, auch Patient ist musikalisch und zeigt akustisch-visuellen Mischtypus. 
Seit vielen Jahren besteht eine allgemeine nerröse Reizbarkeit. Die Stimmung ist meist trübe. Vor $11 / 2$ Jahren war er Zeuge in einem sehr unangenehmen Process gegen einen Verwandten. Pe. wurde nun unruhig und sohlaflos, so dass er ein Sanatorium aufsuchte. Einige Zeit glaubte er, er müsse wegen Falseheides ins Gefängniss, obwohl er sicher keinen falschen Eid geschworen hatte und meinte, er werde deshalb von Polizei und Staatsanwalt gesucht. Nach etwa 6 Wochen trat Heilung ein.

Von jeher konnte er Abends im Bett, bei fehlenden äusseren Reizen, be liebig Personen und Scenen "sehen". Diese "lebenden Bilder" tauchten auf und verschwanden, zeigten aber keine Bewegung. Wenn durch eine Theatervorstellung oder dergl. die Bahnen stärker activirt waren, zogen auch obne sein Zuthun die Scenen noch einmal an ihm vorüber, genau so plastisch, scharf und farbig wie vorher im Theater. Besonders lebhaft waren die Visionen vor einigen Jahren, als er im Geschäft seine Augen stärker anstrengen musste. Die Untersuchung ergab eine Hyperopie mässigen Grades. Als er dann bei der $\Lambda$ rbeit Brille trug, hörten die nächtlichen Spontanvisionen sọfort auf. Die Tăhigkeit, durch Aufmerksamkeitsanspannung Hallucinationen zu erzeagen, blieb unverändert.

Seit etwa 1903 erscheinen ihm, wenn er Gemüthşbewegungen gehabt hat, Abends im Bett die Köpfe der Leute, mit denen er tagüber zu thun hatte, ab und zu auch unbekannte Köpfe.

Ungefähr 1904 acquirirte er einen linksseitigen Mittelohrkatarrh, der chronisch wurde. Einige Zeit nachher begannen die hallucinirten Köpfe und Personen zu sprechen. Die Stimmen waren sehr laut und wurden nur auf dem linken Ohr gehört.

Niemals, auch nicht nach dem Besuch ron Oper und Concert singen oder musiziren die hallucinirten Personen. Patient laann aber ohne Mühe alle gehörten einfacheren Melodien auf dem Clavier ohne Noten spielen.

Im April 1907 regte Pe. sich über seine Frau sehr auf. Nun sah er acht Tage jeden Abend im Bett dio Köpfe stundenlang und hörte anf dem linken Ohr sie laut Verse recitiren oder gleichgültige Dinge sprechen. Er selbst musste oft mitsprechen und antworten. Obwohl der Wirklichkeitscharakter der Hallucinationen sehr gross war - ,jeh sehe und höre es nicht anders, wie wenn ich im Geschäft mit den Leuten verkehre," sagte Patient -, war er stets überzeugt, dass er Trugwahrnehmungen habe und linüpfte keinerlei Schlussfolgerungen daran. Der Schluss auf Sinnestäuschung, meinte er, sei deshalb nicht schwer, woil er nur die Köpfe und nicht die ganze Situation sebe. Ich habe nach dem Vorgang Kandinsky's I) und Störring's' (l. c.) die Beziehungen der hallucinatorischen Bilder "zum Augenschwarz des objectiven Gesichtsfeldes " härifg geprüft. Stets waren sie „in den im gegebenen Moment wahrgenommenen Raum eingeordnet und ron den Bewegungen des Sinnesorgans abhängig" (Störring). Am Tage hörte er keine Stimmen, sah auch keine Bilder, hatte aber zeitweilig den ungewohnten Drang, viel zu

1) Kandinsky, Archiv f. Psych. XI. 
sprechen. Gelegentlich kamen auch Gedanken und Sätze dabei heraus, die er als fremdartig empfand, so dass er sehr erstant war.

Im August 1907 hatte er wieder einen Aerger wegen der alten Processsache. Im Geschäft war er zerstreut, die Stimmung war deprimirt. Er schlief schlecht und hatte eigenthümliche Sensationen in Kopf und Rücken. Einige Male hörte er Nachts in der Stirn seinen Namen rufen und auch andere Worte: ,ich will, was ich will ". Visionen waren gleichzeitig nicht vorhanden. Volle Correctur auch im Augenblick der Hallucination. Nach 3-4 Tagen traten die Erscheinungen nicht mehr auf.

Nun ging es ihm 6 Wochen sehr gut, bis er im October 1907 wieder in einen Familienzwist hineingezogen wurde. Es wiederholte sich die selbe Attacke wio im April und hielt 5 Tage lang an.

Versuche, hallucinirte Köpfe etc. anf ein Blatt Papier zu zeichnen, gelangen nicht. Die Bilder verschwanden leieht, wenn die Aufmerksamkeit abgelenkt wurde, auch fehlt zeichnerische Fertigkeit.

Patient hat stets alle Hallucinationen vollkommen corrigirt und niemals Wahnvorstellungen gehabt, die in irgend einer Beziehung zu den Hallucinationen standen. Depressive Wahnvorstellungen haben überhaupt nur einmal vor $1 \frac{1}{2}$ Jahren kurze Zeit bestanden.

Der ganze centripetale und centrifugale Leitungsbogen der Sehbahn Pe.'s einschliesslich der Erinnerungscentren war von Jugend an übererregbar. Der sensorische Antheil kann bei Körperruhe und Abwesenheit äusserer Reize durch Aufmerksamkeitsanspannung zur Function (Visionen) gebracht werden. Der motorische Antheil bedarf jedoch jedesmal noch einer besonderen Activirung. Bewegte. Visionen werden nur wahrgenommen, wenn gerade Sinneseindrücke mit lebhafter Gefühlsbetonung voraufgingen. Gemüthsbewegungen überhaupt rufen auch passiv Visionen hervor.

Der gesammte sensorische und motorische Leitungsbogen der Hörbahn ist durch einen Mittelohrkatarrh activirt worden. Die Gehörshallucinationen werden meistens associativ durch Visionen ausgelöst. Niemals aber war, selbst in Zeiten grosser Anfregung, der umgekehrte associative Weg gangbar. Die Gehörshallucinationen haben nie Gesichtstäuschungen im Gefolge gehabt.

Die Hallucinationen unterscheiden sich in keiner Weise von realen Sinnesempfindungen. Trotzdem wurden sie durch Controlle und $\mathrm{Zu}$ sammenwirken weiterer Sinnesempfindungen stets corrigirt.

4. Fran 0., 31 Jahre alt, nicht belastet. Stammt aus einer sehr musikalischen Familie, ist selbst musikalisch künstlerisch begabt. Akustischvisueller Typus. Der akustische Antheil wiegt vor. Früher nie krank.

Vor einigen Jahren depressive Verstimmung leichten Grades. Sah in dieser Zeit sehr hänfig die Gesichter der Anwesenden sich in Leichengesichter 
verwandeln, hatte Angstgefühl dabei. Oeftere Remissionen. Heilung nach mehr als einem Jahr.

Geringe Myopie, die nie Störungen verursacht hat; Ohren gesund.

Hat von Jugend an die Gabe bei geschlossenen Augen, wenn die Umgebung nicht zu lebhaft ist und nicht ablenkend wirkt, durch einen Willensact (Aufmerksamkeit) bekannte Personen in realer Deutlichkeit zu sehen. Die Vision bleibt an der Stelle schärfsten Sehens und wandert entsprechend mit den Augenbewegungen. Nach dem Besuch einer Oper und eines Concertes bewe ge $n$ sich etwa einen Tag lang die Figuren, wie sie sich auf Bühne und Podium bewegt haben. Gleichzeitig wird die gehörte Musik - Gesang und Instrumente - hallucinatorisch laut, und zwar in derselben Klangfarbe und Stärke wie bei den realen Empfindungen. Patientin kann ihre Hallucinationen stundenlang andauern lassen. Sowie aber die Aufmerksamkeit abgelenkt wird oder ein stärkerer Sinnesreiz von aussen herantritt, verschwinden die Trugwahrnehmungen sofort. Stärkere Bahnung durch Gesichtseindrücke (Theater ete.), Affecte und Gemüthsbewegungen erleichtern die Auslösung der Hallucinationen.

5. 0., der älteste Sohn der Patientin sah rom 6.-10. Lebensjahr Abends im Bett Blumen durch die Luft fliegen, Automobile und Räder auf sich zufahren, sah Gegenstände im Schlafzimmer grösser und grösser werden. Die Visionen wurden nicht für wirklich gehalten, flössten dem Kinde grosse Angst ein. - Augen und Ohren sind gesund.

In beiden Fällen ist die gesammte sensorische und motorische Sehbahn übererregbar, im Fall 4 ist aber ebenfalls (cf. Fall 3) die motorische Sehbahn die schwerer ansprechbare. Während aber Pe. nie musikalische Hallucinationen hatte, traten sie hier auf associativem Wege sebr leicht auf. Niemals, abgesehen von der Zeit des Depressionszustandes, sind spontane Hallucinationen beobachtet worden. Die Correctur ist selbstverständlich, da die Erscheinungen nur durch einen Willensact (Aufmerksamkeit) hervorgerufen werden.

6. Li., Arbeiter, geboren 1866. Nicht belastet. 1887 erlitt er eine Schwefelkohlenstoffvergiftung und erkrankte an depressivem hallucinatorischen Wahnsinn. Aufnahme in die Psychiatrisohe und Nervenklinik der Universität Leipzig am 23. Februar 1887. Angst, Gehörs- und Gesiohtshallucinationen, Personenverkennung, Schwindelgefühl, grosse Unruhe, traumhaftes Benommensein. Geheilt nach 8 Monaten ${ }^{1}$ ). Nach der Entlassung begann er wieder zu arbeiten. Sehr bald stellten sich Gehörshallucinationen ein. Er musste hinhorchen und arbeitete deshalb langsamer. Seine Arbeitsstelle wechselte er vielfach, hielt nirgendwo lange aus.

Der hallucinatorische Zustand wurde chronisch. Er hörte bald angenehme, bald unangenehme, bald gleichgültige Dinge, die er stets corrigirte.

1) Krankengeschichte ausführlich bei Lau denheimer, Schwefelkohlenstoffvergiftung. Leipzig 1899. 
1896 hatte er Bleikolik. Anfangs 1897 mehrten sich die Gehörshallucinationen, "so dass er ganz in Verwirrung geriet". Ausserdem hatte er lange dauernde "Nachbilder" von Personen, mit denen er gesprochen hatte.

Aufnahme in die Klinik am 9. Januar 1897. Gehör normal, Ohrenbefund normal, geringe Myopie, Fehlen des Geruchssinnes. Intelligenz dem Bildungsgrad entsprechend. Fast ununterbrochen hörte er Stimmen, die ihn sogar oft am Einschlafen hinderten. Selten hatte er für einige Stunden oder gar einen ganzen Tag Ruhe. Anfangs hörte er die Stimmen vorwiegend links, von März $1897 \mathrm{ab}$ auf beiden Ohren. Er behauptete stets das Krankhafte der Stimmen zu erkennen. Doch sei es ,vielleicht eine Art Ahoung, dass die betreffenden Leute zu der Zeit, wo er sie hörte, wirklich etwas von ihm sagen". Ablenkung durch Lesen möglich. Die zahlreichen hallucinatorischen Befehle befolgte er nie. Von Ootober 1898 an nahm er jede bekannte Person oder Situation, die er sich ins Gedächtniss zurückrief, mit realer Deutlichkeit wahr, "so dass er sie zeichnen könnte". Er sah nun auch meist die Personen, deren Stimmen er hallucinatorisch hörte, jedoch in einer gewissen Entfernung, „als ob die Mauer dazwischen wäre" grösserer Entfernung - sah, hörte er hallucinatorisch sie stets laut sprechen. - Die Stimmen sprachen seine Gedanken aus, schjmpften, sagten gleichgültige oder angenehme Dinge. Die Stimmung war meist deprimirt. Krankheitseinsicht bestand stets. Im ersten Moment glaubte er oft an die Wirklichkeit seiner Hallucination, überlegte sich aber dann sofort, dass es sich nur um Sinnestäuschungen handele. Wenn er von Mitpatienten belästigt wurde, häuften sich die Hallucinationen. Dann kam es nicht selten vor, dass er sich seine Correctur der Trugwahrnehmungen von Arzt, Personal und Mitpatienten noch eigens bestätigen liess. Das Wirklichkeitsgefühl der Hallucinationen war immer sehr stark.

Wesentliche Aenderungen im Befinden traten nicht ein. Nach fast zwei Jahren verliess Patient die Klinik und nahm Stellung als Markthelfer an.

Die Hallucinationen dauerten fort, verschwanden oft für einige Tage, waren bald sehr gehäuft, bald seltener, bald sebr laut, bald leise.

15. Mai 1900 Wiederaufnahme wegen gehäufter Hallucinationen. Die Stimmen sprachen wieder in endloser Kette. Diesmal börte er nur seine Angehörigen sprechen und sah sie dabei zuweilen.

"Ich sehe sie aber nicht mit den Angen, denn sie sind auch da, wenn ioh die Augen zu habe. Dass Alles Unsinn ist, weiss ich im mer ganz genau und trotzdem werde ich vor dem Einschlafen manchmal ängstlich." Nie knüpfte Patient irgend welche Sohlüsse an seine Hallucinationen, nie richtete er sich nach den gehörten Befehlen. Die erst sehr mürrische Stimmung warde allmählich besser. Die Hallucinationen liessen nach. Es wurden nur noch ab und zu seine Gedanken laut, die er von einer Tante aussprechen hörte. Spontan traten Gesichtshallucinationen selten auf, meist bewegte Scenen hässlichen Inhalts. Durch einen Willensimpuls (Aufmerksamkeit) vermochte er zu den Stimmen die zugehörigen Visionen zu erzeugen, zu jeder wirklich gesehenen 
bekannten Person deren Stimme hinzu zu halluciniren. - Entlassen nach drei Monaten.

Der Zustand besteht jetzt (1907) fast unverändert fort. Patient arbeitet seit 1900 regelmässig.

Die hallucinatorischen Wahrnehmungen hatten immer genau alle Eigenschaften von wirklichen Sinneseindrücken, anch die Gefühlsbetonung war nicht anders. Die Correctur erfolgte nach der Angabe Li's nur durch logische Schlussfolgerung.

Nach einer schweren Intoxicationspsychose 1887 blieb eine hochgradige Uebererregbarkeit der Hörbahn zurück. Die Hallucinationen betreffen nur die sensorische Bahn, eine Betheiligung des motorischen Antheils des Bogens ist wenigstens nicht nachweisbar.

Eine Bleivergiftung 1896 activirte auch die Sehbahn, in der allerdings bereits während der Psychose 1887 Hallucinationen gewesen waren, so dass anfangs 1897 Visionen sich einstellten (zuerst "Nachbilder"). In der Sehbahn war de: motorische Theil des Leitangsbogens sehr viel geringer betheiligt, als der sensorische. Anfangs erschienen die Visionen als unbewegte Nachbilder, 1900 zuweilen als bewegte Scenen.

Als Lokalzeichen der Hallucinationen machten sich geltend laute oder leise Stimmen, Fjguren als Nachbilder und solche in festbestimmter Entfernung, „als ob die Mauer dazwischen sei!".

Im Gegensatz zu Fall 3 sind die Associationsbahnen zwischen den centralen Gesichts- und Gehörsorganen (Centren) von jedem der beiden aus sehr leicht ansprechbar. Die Gesichtshallucination vermag associativ durch Aufmerksamkeit oder zufällige andere Association sofort eine Gehörshallucination auszulösen und der umgekehrte associative Weg ist ebenso prompt gangbar. Trotz des vollkommenen Objectivitätscharakters und Wirklichkeitsgefühl der Hallucinationen, trotz ibrer Massenhaftigkeit und einer Dauer von 19 Jahren werden sie durch Schlussfolgerungen stets corrigirt.

7. Be., Privatgelebrter, geboren 1858. Eine Schwester geisteslirank. Patient, der nie ernstlich krank war, wurde während seiner Studienzeit eigenthümlich, kam nicht zum Examen, vereinsamte mehr und mehr. Wegen starker Sensationen in den Genitalien und Zehen begab er sich mit 32 Jahren, 1890, in die psychiatrische Klinik. Als Junge hatte er einen barmlosen Ausschlag an den Genitalien. Glaubte; das sei schimpflich, er müsse sich von den Leuten fernhalten. Das Gefübl blieb, als er über die Harmlosigkeit des Ausschlags aufgeklärt wurde und dieser beseitigt worden war. Er war damals sehr menschenscheu, fühlte sich in Gesellschaft Anderer sebr unwohl, sprach möglichst mit Niemand. Er glaubte, es gehe, durch seine trübe Gemüthsstimmung bedingt, von ihm ein Fluidum aus, welches sogar durch Thüren auf Andere einwirke. Ferner hatte er den Gedanken, dass alle Menschen ihm sein 
Leiden ansehen könnten. Er liess sich bald überzeugen, dass kein Grund zu derartigen Gedanken vorhanden war. Die Gedanken drängten sich trotzdem immer wieder auf und hatten dann den Charakter echter Zwangsvorstellungen.

Auf dem rechten Ohr bestand ein alter cbronischer Mittelohrkatarrh. mit reichlicher Secretion. Später war auch ein linksseitiger Mittelohrkatarrh vorhanden.

1895 wurde lautes Sprechen rechts in $13 / 4 \mathrm{~m}$, links in $1 / 2 \mathrm{~m}$ Entfernung gehört. Allmählich nahm die Schwerhörigkeit noch weiter zu.

Um 1895 traten im $\mathrm{Ohr}$ zuweilen eigenthümliche Sensationen wie Flüstern auf. Er hörte aber Anfangs keine Worte, wurde auch von den Erscheinungen nicht beunruhigt.

Etwa um dieselbe Zeit stellte sich das Gefühl ein, dass alle Leute ihn ansähen. Obwohl er sich sagte, dass keine Veranlassung dazu bestehe, wurde er doch verlegen oder ärgerlich, was sich auch im Mienenspiel zeigte. Wenn er in das Café ging und Jemand sah ihn an, trat sofort die peinliche Vorstellung auf, man sehe ihm seine Krankheit an. Oft hatte er auch die Vorstellung, er werde angesehen, ohne dass es der Fall war. Obwohl er sich stets sagte, dass sein Gefühl eine Täuschung sei, wagte er doch nicht aufzusehen, und vertiefte sich am liebsten scheinbar in eine Zeitung. Der unnütze - wie er selbst einsah - Kampf mit den unnötigen Peinlichkeitsgefüblen regte ihn "furchtbar" auf; er kam in "fürchterliche "Stimmung.

Die Flüstersensationen im $\mathrm{Obr}$ dauerten an. Bald kam aber sehr laut das Geräusch zugeschlagener Thüren hinzu. Er konnte nie unterscheiden, ob wirklich eine Thür geschlagen wurde, oder ob er das Geräusch nur hallucinatorisch wahrnahm. Dabei wusste er genau, dass die meisten dieser täglichen, häufigen Wabrnehmungen Gebörstäuschungen waren. Auch als er 1902 das Geräusch einer sehr nahe, 2-3 m binter seinem Rücken heftig zugeschlagenen Thür in Wirklichkeit nicht mehr hören konnte, blieb die Hallucination unverändert fortbestehen. Da aber die Gesichtsempfindung einer zugeworfenen Thür oft bei ihm die Gehörsempfindung hallucinatorisch auslöste, war er häufig im Zweifel über die Realität dieser Hallucination. Ausser diesen Geräuschen hörte er etwa seit1897 besonders Abends Stimmen. Die sehr lauten Stimmen liessen in derHauptsache seine Gedanken über die Zwangsvorstellungen laut werden, sprachen von den sexuellen Erregungen, von seiner "Willensrichtung". Als er sich mit französischen Sprachstudien beschäftigte, börte or zuweilen auch Wortwendungen, die ihn besonders interessirt hatten.

Im August 1900 hörte er einmal ausser anderen Dingen: "Welche Richtung der Wille?

Mich sozusagen verstecken und verkriechen vor den Leuten,

Durchaus zu abstrahiren von allem Connex mit den Menschen.

$\mathrm{Zu}$ schauen und zu lauschen immerdar, ob man mir dann auch nicht etwas Böses zufügt (niedrig über mich denlit).

Alles vordirbt sich mir durch meinen Unwillen."

Nach Mittheilung des Patienten giebt die Aufzeichnung nicht den Wortlaut der Hallucinationen, wohl aber im Wesentlichen das Gehörte wieder. 
Von dem subjectiven Charakter seiner Sinnestäuschungen war Patient stets überzeugt, wenn er irgend eine Controlle mit anderen Sinnesorganen oder associativen Ueberlegungen ausüben konnte. Jedenfalls aber hatten bei ihm die hallucinatorischen Empfindungen völligen Wirklichkeitscharakter.

Patient ging jeden Monat einmal aus und trank einige Glas Bier. Da er ungemein wenig vertrug, stand er oft bei der Heimkehr deutlich unter Alcoholwirkung. Nun hörte er fast regelmässig den einen Portier, der ibm öffnete, eine despectirliche Aeusserung thun: "Sie kommen recht spät heim", "Sio haben wohi heute gekneipt", ganz gleich ob der Portier nur guten Abend sagte oder überhaupt nicht sprach. Entweder wurde Patient gleich heftig oder: er beschwerte sich am nächsten Morgen bei den Aerzten. Diese Hallucination erkannte er nie als solche, liess sich auch nicht überzeugen.

Patient war aussecordentlich leicht in tiefen hypnotischen Schlaf zu bringen. Die Hypnose wirkte stets für ein bis drei Tage derart, dass Zwangs- vorstellungen und Hallucinationen nabezu verschwanden. Das Krankheitsbild blieb bis 1904 unverändert, dann verliess Patient die Klinik. 1905 starb er an einer acuten körperlichen Krankheit.

Der Fall bietet besonderes Interesse deshalb, weil bei einem Psychopathen, der in der Hauptsache an Zwangsvorstellungen litt, im Anschluss an eine chronische Erkrankung des peripheren Organs corrigirte Hallucinationen auftraten, die zum Theil zu den Zwangsvorstellungen in Beziehung standen. Die gesammte sensorische Hörbahn und noch transcorticale Bahnen und Centren (Gedankenlautwerden) sind übererregbar. Der motorische Theil des Leitungsbogens ist dagegen nie nachweisbar in Mitleidenschaft gezogen worden. Das Lokalzeichen der peripheren Antheile der Bahn, die überlaut erklingenden Geräusche und Töne, war stark ausgeprägt.

Die Hallucinationen hatten, wie Patient stets behauptete, die Realität von wirklichen Sinnesempfindungen, ihr Gefühlston war ebeuso lebhaft. Trotzdem corrigirte er stets durch die Controlle der anderen Sinne und durch Schlussfolgerungen selbst bei Aerger und stärkeren Gemüthsbewegungen. Unter dem Einfluss einer mässigen Alcoholvergiftung aber war es ihm unmöglich, die Hallucinationen als solche zu erkennen. Er deutete sie wahnhaft und die Wahnidee war uncorrigirbar.

Sehr bemerkenswerth ist auch, dass der tiefe hypnotische Schlaf allein ohne fremde Suggestionen im Stande war, Zwangsvorstellungen und Hallucinationen für kurze Zeit fast ganz zu unterdrücken.

8. Ack., geboren 1860, Fabrikmeister. Mutter nervenkrank, Bruder des Vaters geisteskrank, Bruder an Epilepsie gestorben. Patient konnte sehr gut rechnen und hatte ein sehr gutes Zahlengedächtnis. Schreiben und viele andere Dinge lernte er schwerer. Sehr ausgesprochener visueller Typus. Lernt Wort- 
reihen durch optischen Eindruck leicht. Sehr schwer lernt er nur gehörte Wortreihen, kaum solche, die er nachspricht ${ }^{1}$ ).

1889 Bleivergiftung, 4-5 Jahre Lähmung des rechten Armes. 1897 und 1898 arbeitete er mit einem Bronzepulver, das er "Aluminiumgold" nennt. Die Haare seien davon ganz verfärbt worden, das Pulver sei fortwährend wie Schwefel in der Athmungsluft gewesen.

1898 begann er auf allen Sinnesgebieten zu halluciniren, er war völlig verwirrt und hatte die Idee, man verfolge ihn. Der Zustand besserte sich bald, so dass er wieder arbeiten konnte. Von nun an aber hatte erGehörstäuschungen, die bis heute andanern. Er erkennt sie stets als Täuschungen. In den ruhigsten Zeiten besteht nur ein Klingen in den Ohren, als wenn etwas auf Metall aufgeschlagen wird. Dieses Geräusch verlässt ihn nie. Die nächste Steigerung ist Maschinensausen. Tritt in guten Zeiten nach dem Genuss von wenig Alcohol auf.

1903 hörte er sehr viele Stimmen. Er versuchte durch Nachfragen bei seinen Arbeitsgenossen immer wieder, sich von dem hallucinatorischen Charakter der Wahrnehmungen zu überzeugen. Nach etwa sechs Wochen war er so weit geheilt, dass nur noch das Klingen andanerte.

1905 begannen wieder ohne äussere Veranlassung die Stimmen ihn zu belästigen. Sie wurden schliesslich so lebhaft und zablreich, dass er gelegentlich doch in Zweifel kam, ob das alles nur Sinnestä̈uschungen seien. Er ging oft zu den Arbeitern und Vorgesetzten, um sich zu erkundigen, ob sie wirklich dies und das gesagt hätten. Einige Mal kam es sogar vor, dass er irgend einen zugerufenen Auftrag befolgte und erst bemerkte, dass es eine Hallucination gewesen war, wenn er etwa dadurch einen Fehler in der Arbeit gemacht hatte oder die sprechende Person nicht anwesend gewesen sein konnte. Kurze Ruhe zu Hause und geeignete Medication brachten in wenigen Wochen wieder volle Arbeitsfähigkeit und Aufhören der Hallucinationen.

Eine neue schwerere Attacke begann obne wesentliche äussere Veranlassung im December 1906. Patient musste die Arbeit aussetzen und trat den üblichen Gang zum Ohrenarzt an, der wie bei vielen früheren Untersuchungen normale Verhältnisse des peripheren Organs feststellte.

Die Stimmen waren die ron Arbeitern seiner Fabrik, selten die von fremden Leuten. Sie sprachen oft von der Fabrikation, vielfach aber auch andere Dinge: „Du wirst jetzt in der Lotterie gewinnen". Es ist Geld für dich gespart, es liegt da; nächstens kannst du es holon". Der Grund für diese Hallucinationen war der Umstand, dass Patient eine Summe in Raten zurückzahlen musste, was ihm schwer fiel. Ueber diese Hallucination lachte er meist und hielt die Stimme nie für real. Am besten hörte er sie, wenn alles ruhig war und ging daher jeden Tag in den Wald. Aber auch in die Unterhaltung drängten sich die Stimmen hinein und hörten selbst im Strassenlärm nicht ganz auf. Er wurde völlig schlaflos, hatte geringen Appetit,

1) Herr Privatdocent Dr. Brahn hatte die Liebenswürdigkeit, den Ack. eingehend auf seinen psychologischen Typus zu untersuehen. 
magerte stark ab, klagte über Stirn- und Nackenkopfschmerzen. Unter der Medication von täglichen Bädern und Veronal besserte sich der Zustand langsam mit vielen Recidiven. An den schlimmsten Tagen im Januar 1907 hörte er den ganzen Tag die Stimmen alle seine Gedanken aussprechen, fragen, selten schimpfen, und musste ihnen antworten. Er las nicht, weil er seine Gedanken nicht bei der Sache habe. Gelesenes wurdo nicht nachgesprochen. Trotz aller Correction war die Stimmung dann deprimirt. Wenn es etwas besser war und die Stimmen wenigstens zeitweilig, wie bei der Unterhaltung in der Sprechstunde aufhörten, musste er die hallucinirten Worte nach sprechen. "Das Nachsprechen kommt ganz von selbst."

Zu Zeiten, wo nur in der Waldruhe die Stimmen von Geld und von Arbeit sprachen, hatte er etwas „wie einen Hauch auf der Zunge und ein Bewegungsgefühl in derselben, brauchte aber nicht zu sprechen. Nun ging der sonst sehr starke Wirklichkeitscharakter der Stimmen verloren, sie wurden "fremdartig", obwohl der Klang der ihm bekannten Stimmen sich nicht änderte. In schlimmeren Tagen hatte er immer die anderen Sinnesorgane und die Logik zur Correctur anwenden müssen. Das war jetzt nieht nöthig. Zu dieser Zeit lernte er im Walde einen Mann kennen, der selbst krank war, und einige Mal mit ihm über seine eigene Krankheit sprach und auch dabei von Ack's Krankheit erfuhr. Der Mann machte unwillkürliche Bewegungen mit 'dem Munde "wie Sprechen". Ihn hörte Ack in der Folge hallucinatorisch sehr viel.

Wenn ich in diesem Zustand einen schwachen faradischen oder galvaniseben (0,3-0,6 MA) Strom 1--2 Minuten durch den Kopf gehen liess, Elektroden auf beiden Ohren oder Stirn und Nacken, hänften sich bald für den Rest des Tages die Hallucinationen. Patient musste wieder die hallucinirten Worte nachsprechen. War die Anode auf das linke Obr gesetzt worden, waren die Stimmen nacher vorwiegend links. Anode auf dem rechten $\mathrm{Ohr}$ machte keine Besonderheiten. Einzelne oder zahlreiche Schliessungen und Oeffnungen von Anode oder Kathode verursachten während der Sitzung nur unangenehmes Knacken im Ohr. Der Genuss von ein bis zwei Glas Bier hatte dieselben Folgen wie der elektrische Strom.

Im Laufe des März und April gab es gewöhnlich im Anschluss an geringen häuslichen Zwist fast jede Woche für ein bis drei Tage Versehlimmerungen. Mit Zunahme und Verstärkung der haliucinatorischen Erscheinungen wurde alle Mal auch der Schlaf wesentlich schlechter und der Appetit geringer. Im Mai erlitt er einen leichten Unfall, der auf die Hallucinationen keinen wesentlichen Einfluss ausübte.

Von Ende Mai ab liessen die Hallucinationen stärker nach, alle Functionen hoben sich, nur der Schlaf liess zu wünschen übrig. Selten sprachen die Stimmen noch seine Gedanken aus. Andere Dinge hörte er nicht mehr und nachzusprechen brauchte er nicht. Selten war es wie ein $\mathrm{Hauch}$ auf der Zunge.

Im Juni hörten die Stimmen auf zu sprechen, das metallische Klingen aber blieb bestehen. Von Mitte Juni arbeitete Patient wieder. Die regelmässige Thätigkeit fiel ihm schwer. 
Ende Juli 1907 batte er eine leichte Erkältung und Ziehen in den Beinen. Er erhielt zum Einreiben Kampherspiritus. Sofort nach der Einreibung stellte sich das Stimmenhören wieder ein und die Nothwendigkeit nachzusprechen. In der Nacht hatte er "Verfolgungsträume, ohne dass er eigentlieh schlief". Die vermehrten Hallucinationen und gleichzeitigen Kopfschmerzen hielten zwei Tage an. Im August 1907 strengte ihn das Arbeiten sehr an. Nach einigen heissen Tagen war er matt. Nun wurden Gedanken, dio sich auf den Beruf und auf die Krankheit bezogen, laut. Es waren „nicht gerade Stimmen, welche die Gedanken wiedergaben, sie waren aber auch anders als einfache Gedänken".

Anfangs October 1907 stellte sich eine leichte pleuritische Reizung und schmerzbafte Periostitis am Schienbein ein. Patient hustete, hatte Kopfschmerz und schlief auch mit Veronal nicht. Gleichzeitig wurden wieder Gedanken fast laut und nach den Gedanken eine Antwort. "Es wäre doch besser, wenn er arbeitete, er ist nicht so krank. Mit den Schmerzen geht es nicht." "Wir Deutsche fürchten Gott und sonst nichts auf der Welt! Wer hat das doch gleich gesagt?!" Vereinzelt kamen auch "Stimmen". Als er in diesen Tagen den Mann sah, der im rergangenen Winter mit ihm über seine Krankheit geredet hatte, hörte er ibn sprechen, sowie er die Mundbewegungen sah. Dabei wusste Ack genau, dass er in Wirklichkeit in der betreffenden Entfernung nichts hören konnte. Wenn er ganz in der Nähe dieses Mannes war, hörte er ihn Dinge sagen, die den Patienten blamiren konnten. Einmal stellte sich heraus, dass die wirklich gesprochenen Worte den von Ack gehörten ähnlich klangen.

Die Erscheinungen schwanden bald. Als er aber wieder anfing zu arbeiten und sich dabei sehr matt fühlte, stellten sich wieder die fremdartigen Gedanken und Gegengedanken ein, die beinabe, aber doch nicht ganz, laut wurden. Durch den galvanischen Strom $1 \mathrm{MA}$, zwei Minuten lang die Elektroden auf den Ohren, für $1 \frac{1}{2}$ Tag Nachsprechen und Vorsprechen der Gedanken mit Flüsterstimme.

Nur in den schlimmsten Krankheitstagen klangen die Stimmen laut. Sowie sich das Gefühl des Fremdartigen bei den Hallucinationen einstellte, wurden sie immer leiser.

Bei einem hereditär belasteten Mann wurde durch Bleivergiftung das Nervensystem geschädigt. Acbt Jahre später verursacbte eine Metall- (?) Vergiftung hallucinatorischen Wahnsinn mit Trugwahrnehmungen in allen Simesgebieten. Eine bedentende Uebererregbarkeit der gesammten sensorischen und motorischen Hörbahn (Sprechbahn) and einiger transcorticaler Bahnen blieb zurück.

1. Am leichtesten ansprechbar sind die subcorticalen Antheile. Seit zehn Jahren ununterbrochenes Metallklingen.

2. Das nächste Stadium sind fremdartige Gedanken und Gegengedanken (Kräpelin's Apperceptionshallucinationen).

3. Die Gedanken werden häufig leise vor- oder nachgesprochen. 
4. Leise Stimmen, die befehlen; von der Arbeit sprechen u. s. w. Werden als fremdartig empfunden.

5. Hört die Stimmen, deren Worte auch noch wie ein $\mathrm{Hauch}$ auf der Zunge liegen. Die Wahrnehmungen haben Objectivitätscharakter.

6. Patient muss die hallucinatorisch gehörten Worte und Sätze nachsprechen.

7. Er muss auf das Gehörte antworten und manches nachsprechen.

Jedes dieser Stadien kam völlig rein zur Beobachtung und hielt tagelang bis wochenlang an.

Durch Application eines elektrischen Stromes am Kopf lassen sich leicht die Hallucinationen steigern und das nächst höhere Krankheitsstadium erzielen. Wird die Anode auf das linke ohr gesetzt, wiegen die Stimmen auf der linken Seite vor.

Im Stadium 1-4 haben die hallucinatorischen Empfindungen einen "etwas andern" Charakter wie Sinnesempfindungen. Die Gehörsempfindungen im Stadium 1, 3, 4 sind nicht ganz wie mit den Ohren gehört. Ausserdem ist die Gefühlsbetonung eine andere. Das GefühI des Fremdartigen begleitet jede dieser hallucinatorischen Wahrnehmungen.

Die Hallucinationen der Stadien 5-7 haben dagegen Objectivitätscharakter und die Gefühlsbetonung normaler Sinneseindrücke. Sie werden mit Hilfe der andern Sinne und durch Ueberlegungen (Sehen, Nachfragen etc.) trotzdem corrigirt.

$\mathrm{Zu}$ gewissen Zeiten löste der Gesichtseindruck eines sprechenden (den Mund bewegenden) Mannes Gebörshallucinationen aus. Kam er gleich darauf in die Nähe und hörte ihn wirklich, so erfolgte eine illusionäre Umwandlung des Gehörten.

Obwohl ein fortwährender Reizzustand der peripheren (subcorticalen) Bahnen besteht und sich in Metallklingen äussert, erfolgt nicht von hier aus die Ausbreitung der Symptome bei Verschlimmerungen. Im Gegentheil in den entferntesten transcorticalen Gebieten liegt der Ursprungsort. Zuerst fremdartige Gedanken (Wortbilder in Sätzen), dann die Wortklangbilder dazu. Erst bei weiterer Steigerung einzelne imperative und andere Worte, die immer lauter klingen. Darauf anfangs eben angedeutetes Mitklingen der Wortbewegungsbilder, das erst in den schwersten Tagen Nachsprechen und Antworten wird.

Ein dentliches Wandern von den Centralgebieten Flechsig's (Gedanken) zur Peripherie (Bewegung der Zunge und des Kehlkopfs, laute Stimmen). Oder anders ausgedrückt: der Weg geht von den compli- 
cirtesten psychischen Gebilden zu den einfacheren Vorgängen. Rückläufiger Weg:

Auch die Localzeichen sind gut ausgeprägt. Betheiligung der peripheren Hörbahn zeigt sich in den immer lauter werdenden Stimmen, die der peripheren Sprechbahn in den Bewegungsempfindungen der Zunge, die der Centralgebiete in den fremdartigen Gedanken, die nicht als eigentliche eigene Gedanken empfunden werden.

9. Ge., 29 Jahre alt, Kutscher. Atrophia nervi optici beiderseits. Hat fast ein Jahr lang alle Gegenstände in besonders satten, leuchtenden Farben gesehen. Dann begann er schlecht zu sehen. Die Untersuchung der Augen ergabAtrophia nervi optici. 1/2 Jahr später war das Sehvermögen stark herabgesetzt. Nun begann er gegen Abend Blitze, Funken, Flammen zu sehen, die „besonders schön leuchteten". Später sah er bei Horizontallage Kavallerieattacken, Pferderennen, militärisches Exerciren. Farben und Figuren waren übertrieben leuchtend bezw. plastisch. Patient corrigirte alle Halluoinationen, weil sie in seiner Umgebung unmöglich Wirklichkeit sein könnten. Ich habe den Kranken nur einige Wochen gesehen und weiss nichts über den weiteren Verlauf der Krankheit,

10. In einem anderen unvollkommen beobachteten Fall von Hypophysentumor, der frühzeitig das Chiasma opticum zerstörte, bestanden bald nach Beginn der Sehstörung einige Wochen lang Gesichtshallucinationen, Funken, Feverräder, die nicht besonders lebhạt waren und als fremdartig gefühlt wurden. Mit dem Fortschreiten der Grundkrankheit verschwanden die Visionen.

11. Frau S., 27 Jahre alt. Cyclitis anf dem rechten Auge. Etwa ein Jahr nach Beginn der Erkrankung stellten sich Hallucinationen ein, die nur rechts wahrgenommen wurden und zwar nur bei geschlossenen Augen. Die hallucinirten Gegenstände waren Köpfe von Bekannten, Flammen, Linien, Funken. Sie unterschieden sich von normalen Gesichtsempfindungen durch die „unnatürlich satten Farben" und dadurch, dass sie stets "mitten vor dem Auge" waren. Mehr als $1 \frac{1}{2}$ Jahr bestanden die Visionen ganz monoton fort und wurden stets als etwas Fremdartiges gefühlt and corrigirt.

In allen drei Fällen ist die Sehbahn von ihren peripheren, sensibjen Theilen aus activirt worden. Der motorische Antheil des Leitungsbogens war bei 9 stärker, bei 10 und 11 kaum betheiligt.

Die Correctur erfolgte bei 9 durch die Controlle der anderen Sinnesorgane über Aufenthaltsort und Umgebung, bei 10 und 11 auf demselben Wege und durch das Fremdgefühl der Hallncinationen. Ge. und $\mathrm{S}$. hatten charakteristische Lokalzeichen der peripheren Entstehung ihrer Hallucinationen, die leuchtenden satten Farben der Hallucinationen. 


\section{Art der Erkrankung.}

Die Analyse meiner 11 Fälle zeigt, dass es sich nicht um starre, unveränderliche Formen handelt, sondern um Erscheinungscomplexe, die sehr bedeutender Ausbildung und Rückbildung fähig sind, ohne von ihrer Eigenart zu verlieren.

Einer ätiologisch oder klinisch einheitlichen Gruppe gehören nun die beschriebenen und anderweitig publicirten analogen Fälie nicht an. llanche von ihnen ragen direct "bis in die Breite der Gesundheit" (Cramer, Parish ${ }^{1}$ ), von meinen Fällen Bö., Ha., O., Fr. S.

Man schreibt gern productiven und reproductiven Künstlern die Fähigkeit zu, sich Melodien, Tonfolgen, Farben, Figuren ohne adäquaten, äussern Reiz sinnlich vorzustellen. Nachforschungen, die ich anzustellen versuchte, ergaben, dass nur ein kleiner Theil der befragten Künstler diese Gabe hat ${ }^{2}$. $\mathrm{Zn}$ dieser Klasse gehören auch Fr. O. und Pe. in früheren Jahren. Bei ihnen möchte ich aber doch eine pathologische Uebererregbarkeit der visuellen Centren annehmen, da in diesen später Krankheitserscheinungen secnndär einsetzteu.

Bei Ack. und Li. war Geisteskrankheit zweimal, bez. einmal Phase des chronisch-hallacinatorischen Zustandes.

Bes. Hallucinationen waren zum Theil hallucinations obsédantes. Er litt aber auch an Gehörshallucinationen, die zu seinen Zwangsvorstellungen keine Beziehung hatten.

$O b$ es nun berechtigt ist, eine psychologisch Krankheitsform abzugrenzen, die als Hallucinosis simplex etwa dem hallucinatorischen Wahnsinn oder der Hallucinosis Wernickes gegenüber zu stellen wäre, bleibt abzuwarten. Das vorhandene publicirte Material reicht dazu nicht aus. Meine Fälle, die meisten Fälle Uhthoff's, der Fall Probst's u. A. würden sich einer derartigen mehr psychologisch abgegrenzten Form ohne Weiteres einordnen. Doch erscheint mir der Werth der Abgrenzung recht problematisch.

$\mathrm{Zu}$ den Geisteskrankheiten gehören die von mir beschriebenen Bilder nicht.

Für den empirisch historischen Begriff der Geisteskrankheit muss ich daran festhalten, dass der Kranke nicht dauernd über seiner

1) Parish, Trugwahrnehmungen. 1894.

2) Doch habe ich oft den Eindruck gehabt, als seien hier die Grenzen nicht scharf zu ziehen, als müssten bei vielen Künstlern bestimmte Empfindungsund Erinnerungscentren besonders feste und besonders leicht ansprechbare Verbindungen haben. Kül pe und seine Schule leugnen bekanntlich überhaupt den qualitativen Unterschied zwisohen Empfindung und Erinnerung. 
Krankheit stehen darf; dass vielmehr "Geisteskrankheiten Allgemeinerkrankungen des Gehirns (Wernicke) sind.

Krankheiten aber, die als einzige psychische Erscheinung stets corrigirte Hallucinationen, also Reizerscheinungen, in einem oder zwei Sinnesgebieten anfweisen, sind Herderkrankungen des Gehirns. Das Gegenstïck dazu bilden die Herderkrankungen, welche ohne nachweisbaren, anatomischen Befund centrale A us fallserscheinungen machen, wie Aphasie bei Carcinomatose, Typhus etc. (Oppenheim 1 ).

\section{Aetiologie.}

Sehr verschieden ist die Aetiologie meiner Hallucinosen: Intoxicationen, Infectionskrankheiten, Ohreiterung, Atrophia nervi optici, Cyclitis, Tumor, angeborene Anlage. Alle diese Schädlichkeiten baben an einem bestimmten Theil des Leitungsbogens angegriffen und von hier aus bahnend, vorbereitend gewirkt. Sie haben die Bahnen und Centren in einen Zustand von Uebererregbarkeit gebracht, sind aber selbst zum Theil schon unwirksam geworden, als die eigentliche Hallucinose beginnt, zum Theil überhaupt nicht im Stande, die Hallucinationen direct hervorzurufen. Viefach sind noch auslösende Momente nöthig. Nach Pick genügt bei Disponirten zur Auslösung oft schon der leiseste Anstoss. Bereits $\mathrm{Jolly}^{2}$ ) hält die "Hyperästhesie der Sinnesbahnen" für eine wichtige Vorbedingung.

Experimentell nach Jolly's Vorgang vermochte ich durch relativ starke elektrische Ströme bei Ha. und Ack, weder vom Sinnesorgan aus, noch durch diffuse Reize von verschiedenen Stellen des Kopfes aus direct die Centren zur pathologischen Function zu bringen. $1 / 4-1 / 2$ Stunde später sind die Vorgänge in den Zellen der gereizten Centren im Stande, Hallucinationen zu erzeugen. Alkohol ruft zix manchen Zeiten sehr rasch die Hallucinationen hervor oder verstärkt die vorhandenen. Leider ist auf diesem Wege klinisch nicht zu entscheiden, ob hier pathologische Stoffwechselvorgänge in den Zellen die Hallucinationen erzeugen oder ob in dem. übermässig gereizten Leitungsbogen bereits die normalen Vorgänge dazu genügen.

Dass gewisse Gifte in bestimmter Dosirung umschriebene Gebiete im Gehirn direct angreifen, ohne Allgemeinerscheinungen hervorzurufen, ist erwiesen. Santonin macht Gesichtshallucinationen, Hyosein lähmt gleichzeitig motorische Functionen und verursacht Gehörstäuschungen, lange bevor weitere centrale Erscheinungen sich zeigen.

1) Oppenbeim, Lehrbuch 1905.

2) Jolly, Archiv f. Psych. Bd. IV. 
Dass erkrankte Bahnen besonders leicht einer Giftwirkung unterliegen, hat auch Kandinsky gezeigt.

\section{Localzeichen.}

Zahlreiche hallucinatorische Zustände weisen je nach Betheiligung bestimmter Gebiete und nach dem einen oder anderen vorwiegenden Angriffspunkt der verursachenden Schädlichkeit Localzeichen (Localcolorit, Localton) der hallucinatorischen Empfindungen auf. Liegt die Ausgangs- oder Angriffsstelle in der peripheren Hörbahn, kann die Hallucination im Flüsterton gehört werden (Be., Ack.). Weit häufiger ist beschrieben worden, dass die gequollene Bohne im Ohr, auch die Otitis mit sehr auten Hallucinationen einhergehen. Von meinen Fällen Bö., Ha., Be., Ack. Ich beobachtete einen Paranoiker, der Gehörshallucinationen und Sensationen im rechten $\mathrm{Ohr}$ hatte. Er bohrte mit allerlei Instrumenten im Ohr und jedesmal, wenn er eine Verletzung mit Entzündung des Gebörsganges zu Wege gebracht batte, wurden die Hallucinationen unerträglich lant. Als Localzeichen bei Betheiligung der Sprechbahn können Bewegungsempfindungen in Zunge, Kehlkopf, Lippen auftreten. Ack, Probst's Fall. Ziehen ${ }^{1}$ ) erwähnt, dass ein Hallucinant Kehlkopfbewegungen fühlte, als ob es sich um das Wort Vatermörder handelte. (Besonders feine Empfindung oder wahnhafte Deutung?)

Bei Affectionen der peripheren Sehbahn können besonders leuchtende, farbensatte, überplastische Erscheinungen hallucinirt werden. Ich kenne auch Tabiker mit Sehnervenatrophie, die bis zu zwei Jahren vor der deutlichen Herabsetzung des Sehvermögens alle Gegenstände in unnatürlich intensiven Farben and Grenzen sahen.

Dahin gebören auch einige Fälle von $\left.\mathrm{Uhthoff}^{2}\right)(1,3,9$.$) , in$ denen Skotom und Atrophie in deutlichen Localzeichen den Einfluss und die Art der peripheren Ansgangsstellen zeigen.

Centrale Localzeichen. Die Fälle von $\mathrm{Pick}^{3}$ ), Hoches), Hen-

1) Zieben, Physiolog. Psychologie. 5. Aufl, 1900.

2) Uhthoff, Beiträge zu den Gesichtstäuschungen bei Erkrankungen des Sehorgans. Monatsschr. f. Psychiatrie. Bd. V. 1899.

3) Pick, Beiträge zur Lehre von den Hallucinationen. Neurol. Centralblatt. Bd. XI. 1892. -- Derselbe, Hallucinationen in pathologisch veränderten Mechanismen. Wiener klin. Wochenschr. 1905.

4) Hoche, Doppelseitige Hemianopsia inferior etc. Archiv f.Psychiatrie. Bd. XXIII. 1891. 
sehen 1), Albrecht ${ }^{2}$ ) zeigen Hallucinationen in unvollständigen Figuren und verstümmelten Worten. Bei Hoche's Patientin war die Hemianopsie wahrscheinlich functionell. Es handelt sich um ein eigenartiges Localcolorit bedingt durch Ausfallserscheinnngen in dem einen Theil (Sinnescentrum) und Reizerscheinungen in anderen centralen Theilen (Erinneruagscentren) desselben Leitungsbogens. Es ist möglich, dass die betreffenden Theile vorher besonders fest miteinander verbunden waren.

In vielen Fälien sind Localzeichen und Form der Hallueinationen identisch; jedesmal wenn Angriffspunkt und Sitz der Hallucinationen zusammen fallen. Bemerkenswerth ist Ack. mit seinen Gedanken und Gegengedanken, die gerade eben noch nicht laut werden. Es sind Apperceptions-Hallucinationen (Kräpelin) in Worten, deren Sitz und Angriffsort in transcorticalen Gebieten zusammenfallen. Gleichzeitig besteht ein Localzeichen der Erkrankung peripherer Theile der Hörbahn Metallklingen - unverändert fort ohne Einfluss und Zusammenhang mit dem centralen Process.

Wichtig sind nach dieser Richtung auch die drei Fälle Henschen's mit den anatomisch nachweisharen Reizstellen in der einen Sehsphäre, während die andere in Folge ausgedehnter Zerstörungen Hemianopsie veranlasst hatte. Die Hallucinationen traten nur in den nicht defecten Gesichtsfeldern auf.

Der Leitungsbogen.

Aus Erscheinungsform und Localzeichen lässt sich vielfach mit ziemlicher Sicherheit auf die Ausdehnung und Grösse der betheiligten Bahnen schliessen. Es zeigt sich, dass besonders häufig der ganze sensorische Leitungsbogen von der Peripherie bis zu den Erinnerungscentren befallen ist. Dass die Fälle Royet's ${ }^{3}$ ) mit 140:250 pathologischem Augenbefund und die von Redlich und Kaufmann ${ }^{4}$ ) mit 63 pCt. pathologischem Ohrenbefund mindestens zum Theil ebenso gedeutet werden müssen (Betheiligung der ganzen Bahn, Localzeichen der peripheren Leitung), halte ich für nicht unwahrscheinlich. Selbstverständlich hat Uhth off Recht, wenn er meint, dass die gefundenen peripheren Erkrankungen meist nicht ätiologisch wirksam waren.

1) Henschen, Klinische und anatomische Beiträge zur Pathologie des Gehirns. Upsala. 1890-94.

2) Albrecht, Aphasie und Geistesstörung. Zeitschr. f. Psychiatrie. Bd. 61.1905.

3) Royet, Referat im Neurol. Centralbl. Bd. IX. 1890.

4) Redlich und Kaufmann, Ueber Ohrenuntersuchungen bei Gehörshallucinationen. Wiener klin. Wochenschr. 1896. 
Sehr oft ist der motorische Leitungsbogen ebenfalls erkrankt, wie bei Pe., Frau 0., Ack., S. Dass er allein oder vorwiegend erkrankt sein kann, hat Cramer gezeigt. Nicht ganz beistimmen kann ich Wernicke, der in der motorischen Bahn stets den Ausgangspunkt der Hallucinationen sucht. Mir fehlen $z u$ oft in den Hallocinationen die positiven Zeichen dafür. Wollte man aber auf den Ausdruck und das Localzeichen der Motilität verzichten, so kommt man nicht über den Einwand hinweg, dass schon in der Norm bei verschiedenen Menschen ein beträchtlicher Unterschied in der Wegsamkeit motorischer und sensorischer Bahnen besteht - motorischer, sensorischer Typus. Diese Verhältnisse ändern sich bei Hallucinanten nicht, so weit ich die Dinge üborsehen kann.

Schwierig erscheint mir oft bei Gesichtshallucinationen die Feststellung, ob die motorische Sehbahn betheiligt ist oder nicht. Wenn absolut unbewegliche, im Blickfeld stehende, aber plastische Figuren erscheinen, muss da eine Betheiligung oculomotorischer Centren angenommen werden? Derartige Fälle sind sehr zahlreich: Pe. zuweilen, ferner S., Li.; vergl. auch Kandinsky, Uhthoff und Andere. Am häufigsten wird die Erscheinung bei Hallucinanten beschrieben, die in Folge angeborener Anlage Visionen erzeugen könnes.

Dass in ausgedehntem Umfang zugehörige transcorticale Bahnen einbezogen sein können, lehrt jeder Fall von Gedankenlautwerden. Cramer, Moeli1), Goldscheider ${ }^{2}$ ) u. A. baben gezeigt, dass Gedankenlautwerden auch ohne verbale Vorstellungen vor sich gehen kann.

Zumeist bei Geisteskranken, aber auch bei Vergiftungen, hallucinatorischer Veranlagung, Infectionskrankheiten etc. lässt sich sehr häufig eine Betheiligung peripherer Abschnitte des Leitungsbogens nicht nachweisen. Ob man daraus auf Nichtbetheiligung schliessen muss, erscheint mir unsicher. Nachweisbar können bei diesen Hallucinanten grosse Theile der centralen sensorischen, motorischen und transcorticalen. Bahnen erkrankt sein.

Ohne dass eine Allgemeinerkrankung des Gehirns vorliegt, sind zuweilen Associationsbahnen zwischen zwei übererregbaren Sinnesbahnen in Mitleidenschaft gezogen. (Pe., Li., Frau O.)

Auch wenn in beiden Sinnesgebieten selbstständige Hallucinationen vorkommen, kann die Associationsbahn nur von dem einen Centrum aus gangbar sein. Bei Pe. nur vom Sehcentrum aus, bei Li. dagegen vom Sehcentrum zum Hörcentrum und umgekehrt.

1) Moeli, Der gegenwärtige Stand der Aphasiefrage. Berliner klin. Wochenschr. 1891.

2) Goldscheider, Ueber centrale Lese- und Schreibstörungen. 
Die Gehörshallucinationen können ihren Sitz haben in den:

1. (Vorstellungs-? und) Erinnerungscentren,

2. Stätten der Wortklangbilder,

3. Stätten der Wortbewegungsbilder,

4. Stätten der Hand- ete. Bewegungsbilder (bei Taubstummen),

5. subcorticalen Centren (?).

Die Erregung dieser Centren, die in den erkrankten Leitungsbogen einbezogen sein müssen, kann von irgend einer Stelle desselben aus erfolgen, natürlich auch in einem Centrum selbst liegen ${ }^{1}$ ). Jede Reizstelle kann allein, sie kann mit anderen gemeinscbaftlich wirken.

Ueber die Beziehungen des physiologischen Typus zu den Hallucinationen will ich hier nicht reden.

\section{Leitungsrichtung.}

Zulässig, aber nicht unbedingt nöthig, erscheint es mir, den Mechanismus der Hallucinationen Bö.'s und Ha.'s so zu erklären, dass durch den Reiz der peripheren Erkrankung die ganze sensorische Bahn centripetal erregt wurde. Das Hören von Maschinen oder sausenden Drähten entsteht nach der Formel: Empfinden, Wahrnehmen, Wiedererkennen durch nicht adäquaten Reiz, nur auf Grund der Uebererregbarkeit ganz bestimmter, pathologisch fest verbundener Zellcomplexe. Nach Wegfall des Reizes in den Tuben vermag rasches Sinken des Luftdrucks, oder ein electrischer Reiz vom Ohr aus denselben Erfolg zu erzielen. Dagegen wird man bei denselben Hallucinationen nach Alkoholgenuss, nach stärkerer electrischer Reizung vom Scheitel aus wobl nur an Auslösung von einem centralen Angriffspunkt aus denken dürfen. Somit scheint bei demselben Fall der Weg vorwärts und rückwärts gangbar zu sein. Vermuthlich ganz allgemein: Jeder Angriff an irgend einer Stelle des überempfindlichen Leitungsbogens hat denselben Effect.

Dieselbe Ueberlegung gilt für manche Fälle peripherer Aetiologie

Scheinbar machen die Hallucinanten eine Ausnahme, welche willkürlich etwa Visionen hervorrufen können. Hier scheint immer nur der retrograde Weg vom Erinnerungsbild zur Empfindung gegangen zu werden. Doch erleichtert die Bahnung vom peripheren Organ aus durch voraufgegangene lebhafte Gesichtseindrücke das Auftreten der Hallucinationen. Nicht minder aber sind Affecte wirksam. Zwei Reize, die sicher verschiedene Angriffspunkte haben.

Ack. beginnt seit fast zehn Jahren immer wieder so zu halluciniren, dass die transcorticalen Centren den Anfang machen und von dort aus

1) cf. Binswanger, Lehrbuch. 1904. 
bei Verschlimmerungen ein sehr genau verfolgbarer centrifugaler Weg eingeschlagen wird. Setze ich aber die Anode auf das linke Ohr und lasse einen galvanischen Strom von mehr als 2 M.-A. durchgehen, so werden die Ballucinationen links lebhafter und lauter. Bahnung von der Peripherie, starke Reizung der peripheren Hörbahn (Localzeichen).

Bei abklingendem Delirium tremens lassen sich durch Druck auf die Augen (Liepmann), durch langes Fixiren eines übernahen Gegenstandes (v. Bechterew), bei manchèn Deliranten durch einmaliges oder öfteres Zuflüstern eines Wortes ins Ohr Gesichtshallucinationen erzeugen. Liepmann glaubt an die specifische Aetiologie des peripheren Reizes, Bonhöffer meint, dass die Hinlenkung auf den Gesichtssinn das Maassgebende ist. Ich fand vor allen Dingen sehr grosse individuelle Unterschiede. Am häufigsten gelang es noch durch Druck auf den Bulbus Visionen zu erzeugen, wenn eindeutige Hinweise auf den Gesichtssinn, die am Tage vorher noch wirksam waren, keine Gesichtshallucinationen mehr hervorriefen. Zuweilen gelang es durch Zuftüstern vorher hallucinirter Thiere ("Mäuse", „Schlangen") oder dergleichen die entsprechende Vision zu erzeugen oder dieselben Dinge erscheinen zu lassen, die der Druck auf den Bulbus hervorgebracht hatte. Sehr gewöhnlich Jachte der Delirant dazu, da der Wirklichkeitscharakter des Gesehenen anfing verloren zu gehen. Einmal aber wurde einem Deliranten in diesem Stadium das Wort "Teufel" zugeflüstert. Er hatte auf der Höhe des Deliriums kleine Teufel gesehen. Kaum hatte er das Wort gehört, fuhr er mit entsetzlichem Scbreien, in furchtbarster Angst in die Höhe, schlug sinnlos um sich und konnte nur mit grösster Mühe im Bett festgehalten werden. Nach $1 / 2$ Minute trat Ruhe ein. Er hatte eine grosse, schwarze Figur mit langen Krallen gesehen, die ihn zu erwürgen drohte.

Auch diese Beispiele bringen einen Beweis dafür, dass je nach Krankheitsstadium und Individualität einerseits durch periphere, andererseits durch associative Reizung der übererregbaren Bahn derselbe Effect erzielt werden kann. Directe active und passive Aufmerksamkeit auf das halluciuirende Organ kann zu gleicher Zeit ebenfalls Hallucinationen machen, doch versagt dieser Reiz sehr häufig.

Empfindungs- und Frinnerungscentren.

Schon die Analyse vieler ballucinatorischer Zustände in einem Sinnesgebiet und die sehr versehiedenen Formen des Gedankenlautwerdens weisen zwingend darauf hin, dass in der Grosshirnrinde die Felder für Vorstellung, Erinnerung, Empfindung räumlich getrennt sein müssen (Flechsig). Das oft langsame Fortschreiteu in Etappen vom Geräusch über Wort zum Satz und Gedanken scheinen mir kaum eine andere 
Deutung zu gestatten. Bei Ack. hat hat sich in den zehn Jahren seiner Krankheit der umgekehrte Weg von den Gedanken in Wortbildern bis zum lauten Hören und Aussprechen der Gedanken hundert Mal gleichförmig in langsamster Succession, grossentheils auf räumlich bekannten Etappenstrassen bis zu den peripheren Antheilen der Hör- und Sprechbahn vollzogen. Es bedurfte hier wie in so vielen publicirten Fällen einer gewissen, oft geraumen Zeit, bis die Erkrankung vom Centrum retrograd so weit vorgeschritten war (oder auch umgekehrt), dass die Verbindung zwischen dem hallucinirten Einzelwort und den Vorstellungen hergestellt war.

Ebenso sprechen die vielen sehr verschiedenen Formen von mehr oder minder vollkommener Verschmelzung der hallucinirten mit den wirklichen Sinneseindrücken wenig dafür, dass Erinnerungs- und Empfindungszellen identisch sind oder im gleichen Rindenfeld dicht bei einander liegen (Wernicke). Wenig einleuchtend erscheint mir die Auffassung, dass ein bestimmter Reiz eine Rindenzelle als Empfindungszelle und ein zweiter Reiz dieselbe Rindenzelle als Erinnerungszelle in Bewegung setzt (Störring). Einen Beweis für räumliche Trennung von Empfindungs- and Erinnerungscentrum bilden Fälle Henschen's, die auch anatomisch untersucht worden sind. Die Umgebung der Fissura calcarina war völlig zerstört, trotzdem fanden sich in den blinden Theilen des Gesichtsfeldes Hallucinationen. Da das corticale Empfindungscentrum zerstört war, muss das Erinnerungscencentrum der entsprechenden Hemisphäre erhalten geblieben sein. Das Erinnerungscentrum lässt sich nur in den Flechsig'sehen Randzonen der Sehsphäre suchen, deren relativ kleine Zahl von Projectionsfasern die Verbindung mit subcortalen Sehcentren vermittelt. Dass dieser retrograde einfache Weg genügt, Gesichtsempfindungen zu erzeugen, will ich nicht behaupten.

Hier rollt sich nun sofort die Frage nach Congruenz der anatomischen und physiologischen Sinnesfelder auf. Wo physiologische Centren genau untersucht werden konnten, sind sie grösser, zum Theil erheblich grösser als die anatomischen. Genauere Daten und die näheren Beziehungen von Anatomie und Physiologie der Rindencentren sind noch ganz ungeklärt'1).

Gefühlsbetonung.

Die landläufige Ansicht von der übermässig starken Gefühlsbetonung der meisten Hallucinationen und von dem zwingenden, imperativen

1) Vergl, auch Rothmann's Discussionsbemerkung zum Flechsigschen Vortrag über das Hörcentrum auf der Versammlung mitteldeutscher Psychiater und Neurologen in Leipzig 1907. Archiv f. Psych. 1908. 
Charakter der Gehörshallncinationen gilt für uncomplicirte hallucinatorische Zustände ganz und gar nicht. Sie ist aber auch für die Hallucinationen der Geisteskranken in der allgemeinen Fassung nicht richtig. Die Hallucination hat im Allgemeinen keine andere Gefühlsbetonung als die entsprechende reale Sinnesempfindung. Oft ist sie schwächer, weil die Trugwahrnehmung als nicht real erkannt wird.

Sehr gehäufte Hallucinationen, die corrigirtwerden(Ack., Li.) können in Folge des allgemeinen Krankheitsgefühls eine gedrückte Stimmung erzeugen.

Die willkürlich hervorgerufenen Visionen haben oft angenehme Gefühlstöne (Pe., Fr. 0.). - Im Allgemeinen spielt nicht die besondere Gefühlsbetonung der Hallucination allein die Hauptrolle. Auslösende Affecte, $\mathrm{Zwangsvorstellungen,} \mathrm{Wahnideen,} \mathrm{begleitende} \mathrm{Krankbeitssymp-}$ tome, geben die Stimmungsgrundlage $a b$ und beeinflussen weitgehend die Gefühlsbetonung der Hallucinationen. Be. hatte für die erregenden Unlustgefühle, die seine Zwangsvorstellungen und Hallucinationen begleiten, den subjectiven Ausdruck "Unwillen".

Der selten vorkommende imperative Charakter einiger Gehörshallucinationen Acks. kaun nur auf die vollkommene Realität und das Wirklichkeitsgefühl des betreffenden hallucinatorischen Auftrags zurückgeführt werden. Ein stärkerer oder auch nur veränderter Gefühlston der Hallucinationen gegen die normale Empfindung war nie vorhanden.

Die Hallucinationen der Geisteskranken verhalten sich nicht anders. Der imperative Charakter vieler Gehörshallucinationen findet seine Erklärung in dem Wirklichkeitscharakter der Sinnestäuschung, besonders wenn bei Hallucinanten durch Wahnsinn, Amentia, epileptische Dämmerzustände weitgehende Schädigung des ganzen Gehirns Bewusstseinsveränderungen vorliegen. Oder Wahnriehtung, Schwachsinn, paralytische Kritiklosigkeit etc. bestimmen den zwingenden Einfluss der Hallucinationen.

Ein sehr wichtiger Gefühlston, welcher nur Hallucinationen zukommt, und $z$ war nur solchen, die keine vollkommene Verschmelzung mit normalen Sinnesempfindungen bzw. Vorstellungen eingehen, ist das Fremdgefühl. Die häufigste Entstebungsursache dieses Gefübls wird ein dunkel bewusster Vergleich mit wirklich möglichen und wahrscheinlichen Sinneseindrücken sein. Ich bin aber überzeugt, dass damit die Frage nach der Herkunft des Fremdgefühls nicht erschöpft ist. Mindestens bedarf es der Untersuchung, ob nicht der hallucinatorischen Empfindung direct ein Gefühlston des Fremdartigen anhaften kann ${ }^{1}$ ).

1) Cfr. Ziehen's Ausführungen über Gefülıstöne in seiner physiologischen Psychologie. 
Jedenfalls ruft bei corrigirten Hallucinationen, die das Fremdgefühl nicht haben, der bewusste Vergleich zwischen wirklichen und hallucinirten Sinneseindrücken das Fremdgefühl nicht hervor.

Geisteskranke Hallucinanten haben ungemein oft das Gefühl des Fremdartigen ihrer Sinnestäuschungen. Auch der zwingende, imperative Charakter geht dieser Art der Hallucinationen durchaus nicht ab.

Meine corrigirenden Hallucinanten hatten nur zum Theil das Fremdgefühl. Bei andern unterschieden sich die Hallucinationen durch nichts von normalen Sinnesempfindungen. Bei andern wechselte zu verschiedenen Zeiten die Intensität des Fremdgefühls sehr beträchtlich, $z u$ Zeiten ging es ganz verloren.

\section{Correctur der Hallucinationen.}

Der geisteskranke Hallucinant verwendet das Fremdgefühl nicht, oder höchstens sehr temporär zur Correctur seiner Hallucinationen. Er deutet das Fremdgefühl im Sinne seiner Wahnidee als Zeichen fremder Beeinflussung oder dergl.

Dem corrigirenden Hallucinanten ist das Fremdgefübl ein Zeichen der Irrealität seiner hallucinatorischen Wahrnehmungen. Er weiss aber auch, dass es sich um Sinnestäuschungen handelt, wenn die Hallucinationen vollkommen Wirklichkeitsgefühle und Objectivitätscharakter haben, wenn sie ihm im Augenblick des Entstehens ebenso real sind, wie jede andere Sinnesempfindung (Li., Ack.). Die nachträglich angestellte Analyse des Patienten und die daraus gezogene Schlussfolgerung ist auch nur ein Hülfsmittel. Die Analyse und der correcte Schluss sind nur unter bestimmten Bedingungen möglich. Ist die Hallucination durch active Aufmerksamkeit (Willensact) hervorgerufen worden, so besteht beim Hallucinanten gewöhnlich die Vorstellung, dass es sich bei ihm um ein besonders lebhaftes Erinnerungsbild handelt.

Die Hallucination als solche ist ein Herdsymptom, dessen subjectiver Charakter sofort erkannt und gefühlt oder mit Hälfe weiterer Sinnesempfindungen erschlossen wird.

Den Werth einer realen Sinnesempfindung und ihre Uncorrigirbarkeit bekommt sie erst durch die hinzutretende oder auslösende Allgemeinerkrankung des Gehirns.

Für die liebenswürdige Erlaubniss zur Publication der Fälle Li. (6) und Be. (7), die ich als Assistenzarzt der psychiatrischen Klinik beobachtet habe, danke ich Herrn Geh. Rath Flechsig auch an dieser Stelle. 